\title{
A simplified rate dependent model of forming and wrinkling of pre-impregnated woven composites
}

\author{
A. A. Skordos*, C. Monroy Aceves and M. P. F. Sutcliffe \\ Department of Engineering, University of Cambridge, Trumpington Street, Cambridge, CB2 \\ $1 P Z, U K$
}

\begin{abstract}
A simplified finite element model is developed and validated for the forming/draping of preimpregnated woven composites, incorporating the effects of wrinkling and strain rate dependence. The model development builds upon previous work on simulation of fabric draping using a truss representation of the woven material. Tows are modelled by stiff elastic bar elements, and the non-linear rate dependent shear behaviour is incorporated in elasticviscoplastic elements that follow an appropriate phenomenological constitutive model. Wrinkling due to tow buckling is simulated by allowing the deactivation of tow elements that undergo compressive deformation. The model convergence is tested and its validity is checked against experimental results from the forming of pre-impregnated woven carbon hemispheres. It is found that the model reproduces successfully experimental measurements of shear and wrinkling with a relative error of approximately $4 \%$, while solution times are kept below $60 \mathrm{~s}$ on a conventional PC. These features allow potential iterative use of the model within a process optimisation scheme. The sensitivity of the process outcome to process parameters such as blank holder force and forming speed is investigated.
\end{abstract}

Keywords: A. Fabrics/textiles; C. Finite element analysis (FEA); E. Forming

\footnotetext{
* Corresponding author

E-main address: aas46@,cam.ac.uk

Telephone: +441223364772

Fax: +44 1223332662
} 


\section{Introduction}

Manufacture of advanced composites involves processes of different physical nature, including forming, consolidation or impregnation, and curing in the case of thermosetting matrices. The outcome of each process influences the rest of the manufacturing stages, and feeds through to the design of the composite component, creating complex interdependencies between manufacturing parameters, design constraints and properties of the final part. Modelling of draping and forming of woven material is an essential step in process design and optimisation involving such materials. Research on forming of woven textiles can be classified into two general categories; kinematic models and continuum mechanics-based models. Kinematic models [1-4] are geometrical mapping algorithms that are well developed and implemented in commercial software packages. They treat the woven material as a pinjointed net of inextensible fibres, without including the effect of material properties. Effects such as non-linear shear response, decrimping, wrinkling and slip between tows are ignored. As a result, kinematic models are computationally very efficient but they are only sensitive to the geometry of the manufacturing tool and the initial path of draping. Continuum mechanics approaches usually employ finite element (FE) formulations to solve the forming problem, in which the woven material is modelled as a thin sheet [5-8]. Successful implementation requires the development of appropriate material models that take into consideration mechanical effects relevant to forming. The non-linear character of this type of analysis results in very long solution times which can limit its applicability within a process design/optimisation context where iterative execution of the model is required.

A new class of forming models that constitute truss-based simplifications of continuum models has been proposed recently [9-11]. These models involve modelling of tows of the woven material by using an assembly of pin-jointed elastic bar elements. The shear behaviour of the sheet of material is incorporated in these models either by overlaying a set of shell elements on the assembly of bars $[9,10]$ or by connecting the diagonal nodes of the truss with bar elements that have appropriate non-linear stiffness [11]. Truss-based models allow efficient analysis that incorporates the mechanics of the material, thus offering the potential for use within a process design/optimisation.

Modelling of the viscous behaviour of pre-impregnated woven textiles is an integral part of mechanics-based forming models. Phenomenological continuum models which take into account the viscous or viscoplastic response of the woven textile have been developed [1214]. The material in these models is considered to behave as an anisotropic, incompressible 
viscous fluid or viscoplastic solid, which is inextensible in the tow directions. As this is a purely phenomenological approach, picture frame or bias extension tests are required to estimate of material parameters. Predictive approaches based on analysing the mechanics of woven textiles at a unit cell level have also been proposed $[15,16]$. The analysis of the unit cell can be performed using finite element modelling [15], in which case computationally intensive mesh generation and model solution steps are necessary, or using analytical models [16] that constitute computationally efficient approximations based on the constituent properties and information on the kinematics of deformation. The aforementioned models and their implementations focus on modelling successfully the loading shear behaviour of woven textiles. However, during manufacturing, deformation is not necessarily monotonic. For example, in the case of an uneven double dome geometry, part of the woven material that is draped over the lower dome shears in opposite directions before and after contact with different portions of the tool. This situation arises because when the woven sheet first comes in contact with the higher dome, material in the bias direction shears so that bias strain is positive in the radial direction with respect to the higher dome. When part of this material comes in contact with the lower dome shear occurs in the bias direction so that bias strain is positive in the radial direction of the second dome. As the radial directions with respect to the two domes do not always coincide, some of the material undergoes a shear loading/unloading path.

The work presented in this paper builds upon the draping simulation methodology based on the truss representation of woven materials. The truss-based model proposed in [11] is extended to include:

i. Strain rate dependence in the response of elements of the model controlling shear response

ii. Non-linear response of tows due to decrimping effects

iii. Wrinkling due to buckling of groups of tows

The model requires the phenomenological constitutive relationships which incorporate the elastic-viscoplastic shear response and the decrimping of tows. Analysis of the mechanics of a unit cell leads to an efficient inversion procedure for the estimation of the parameters of the elastic-viscoplastic and of the non-linear elastic material constitutive relationships from bias extension and tensile tests.

Research on manufacturing faults during forming has focused so far on wrinkling caused by excessive shear after tows lock up [17]. In this case, out-of-plane deformation is caused by compression in directions transverse to tows. The model developed in this study accounts for 
a different mode of wrinkling that is manifested as buckling of one set of tows and is observed when compression in the tow directions occurs while the material remains unsheared. This type of wrinkling influences the mechanical response of the produced component significantly, as elastic properties in the direction of buckled tows are governed by their bending stiffness instead of fibre modulus. The methodology used to model this type of wrinkling is based on concepts developed for the modelling of membrane wrinkling [18] and involves an element deactivation/activation strategy. The model formulation is appropriate for implementation in commercial finite element codes, thus exploiting their capabilities in modelling complex geometries and boundary conditions. Hemisphere forming is used to demonstrate and validate the performance of the model and the sensitivity of the process outcome to forming speed and blank holder force is investigated.

\section{Model description}

\subsection{Truss model}

The simplified forming model simulates the woven fabric as an assembly of bars, as shown in Fig. 1. All bars are connected at the corners of the unit cell via pin joints. The sides of the unit cell represent the tows of the textile and the diagonal bars govern the non-linear shear behaviour of the assembly. This arrangement allows the trellising action observed in practice to be reproduced when the effective stiffness of the tow bars is significantly higher than the shear resistance of diagonal bars. Under shear, one of the diagonal elements is in tension and the other in compression.

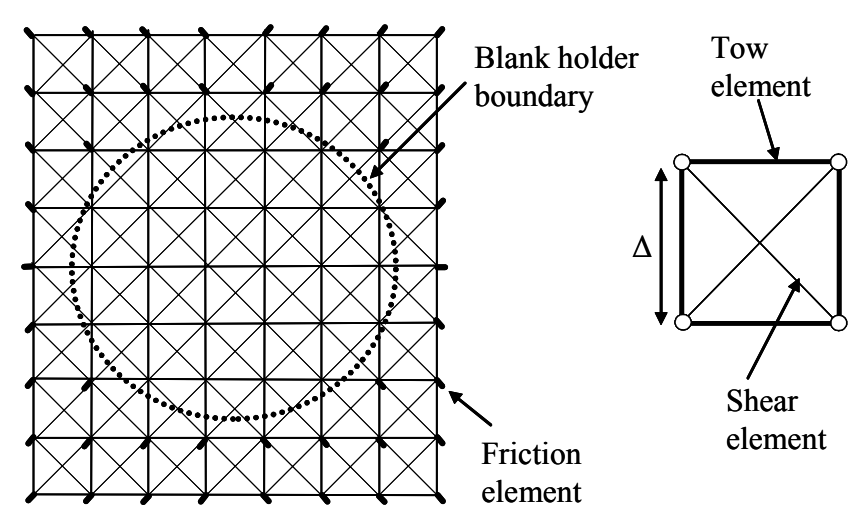

(a)

(b)

Fig. 1. Simplified forming model. (a) Truss assembly. (b) Undeformed unit cell.

The incorporation of wrinkling due to tow buckling is based on an activation/deactivation strategy applied to tow elements. Deactivation is triggered by the existence of compressive 
strain in the element and reactivation by the existence of tensile strain. This simple approach is attractive due to the ease with which it can be implemented across a range of analysis platforms, and is justified by the effective way in which it models wrinkling, as shown in section 4. More sophisticated models which can derive the wrinkled configuration are available in explicit FE codes, but the emphasis of this work is on relatively fast algorithms which can be implemented in an optimisation routine. As noted in the introduction, wrinkling due either to excessive shear or compressive stresses can arise. Here we consider the mode associated with compressive stresses, as these have a more significant bearing on mechanical performance (affecting properties in the tow directions). It is expected that wrinkling associated with shear can be simply correlated with the maximum shear angle. The action of the blank holder on the woven material is incorporated in a simplified but pragmatic way in the model by the inclusion of a group of bar elements connected to nodes in contact with the blank holder. These elements, which are fixed with respect to all degrees of freedom on their free ends, simulate friction via their perfectly plastic behaviour. Their yield stress is

$$
\sigma_{y}=\frac{F_{t o t} \mu}{A_{f} n_{s}}
$$

where $F_{\text {tot }}$ is the total clamping force, $\mu$ is the textile-tool friction coefficient, $A_{f}$ is the cross-sectional area of the element, and $n_{s}$ is the total number of friction elements, which equals the number of nodes in contact with the blank holder. Although this model does not exactly reproduce the usual assumption, that the friction force acts parallel to the direction of the slip velocity (instead acting along the direction of the total displacement of a node), it is felt to be adequate in this application.

The material behaviour of tow and diagonal shear elements follows directly from experimental observations and the operational requirements of the simplified model. Tow elements govern the tensile behaviour of the material in the tow direction, which typically follows a bilinear stress-strain curve, with the initial part corresponding to changes in undulation and tow compression and the second part following the elastic behaviour of the tow $[19,20]$. A simple elastic model is adopted in this approach, neglecting for example coupling between warp and weft tow strains seen in textiles modelling, though in principle this could be incorporated. A non-linear elastic constitutive relationship is used to express this behaviour,

$\sigma_{t}=G\left(\varepsilon_{t}\right)$ 
Here $\sigma_{t}$ and $\varepsilon_{t}$ denote the tow element stress and Green-Lagrange strain respectively, and $G$ is

$G\left(\varepsilon_{t}\right)=E_{\infty} \varepsilon_{t}+\frac{E_{o}-E_{\infty}}{\alpha} \ln \left(\frac{1}{1+e^{-\alpha\left(\varepsilon_{t}-\bar{\varepsilon}\right)}}\right)-\frac{E_{o}-E_{\infty}}{\alpha} \ln \left(\frac{1}{1+e^{\alpha \bar{\varepsilon}}}\right)$

This expression results in linear elastic behaviour with effective modulus $E_{o}$ at very low strains and linear elastic behaviour with effective modulus $E_{\infty}$ at high strains. The transition from the low modulus region to the high modulus region occurs at a transition strain $\bar{\varepsilon}$ and has breadth proportional to $1 / a$. The incorporation of wrinkling due to tow buckling is implemented by deactivating tow elements that are under compression. This strategy is equivalent to

$$
G\left(\varepsilon_{t}\right)=0, \quad \varepsilon_{t}<0
$$

The one-dimensional constitutive model of shear diagonal elements is purely phenomenological. It can be represented as shown in Fig. 2(a). It comprises a Maxwell element with a non-linear dashpot with strain dependent viscosity $\eta$, connected in parallel with an elastic-plastic element of constant yield stress $\sigma_{o}$ and allows for viscous behaviour in both the elastic and plastic phases of deformation.

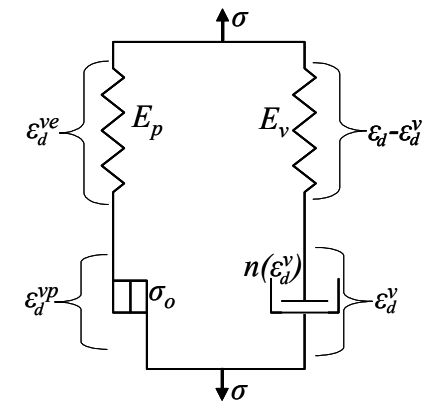

(a)

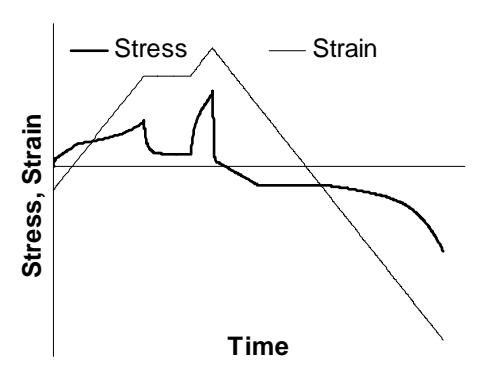

(b)

Fig. 2. Constitutive model of the shear diagonal element. (a) Schematic representation. (b)

\section{Response in loading-relaxation-reloading-unloading.}

The dependence of viscosity on strain is selected so that it reproduces experimental observations of the shear behaviour of woven textile composites. A sequence of three mechanisms occurs as shear strain increases: (i) flexural deformation of tows; (ii) friction controlled tow rotation at crossover regions and; (iii) lateral compression of the tows [21, 22]. It follows that the material is more compliant at low strains when bearing-type contact effects dominate deformation, whereas shear resistance increases rapidly at higher strains due to lateral compression and intra-tow friction which eventually cause lock up of the textile structure. The strain is decomposed into viscoelastic and viscoplastic contributions as 
$\varepsilon_{d}=\varepsilon_{d}^{v p}+\varepsilon_{d}^{v e}$

The material behaves as a viscoelastic solid when the viscoelastic strain is below the yielding strain or when the stress and strain rate have opposite signs, i.e. $\left|\varepsilon_{d}^{v e}\right|<\sigma_{o} / E_{p}$ or $\sigma \dot{\varepsilon}_{d} \leq 0$. In this regime the material behaviour is

$\sigma+\dot{\sigma} \frac{\eta}{E_{p}}=E_{p} \varepsilon_{d}^{v e}+\frac{E_{p}+E_{v}}{E_{v}} \eta \dot{\varepsilon}_{d}$

When the viscoelastic strain exceeds the yield limit and the sign of stress is the same as the sign of strain rate $\left(\left|\varepsilon_{d}^{v e}\right| \geq \sigma_{o} / E_{p}\right.$ and $\left.\sigma \dot{\varepsilon}_{d}>0\right)$ the material behaves viscoplastically and

$\sigma+\dot{\sigma} \frac{\eta}{E_{v}}= \pm \sigma_{o}+\eta \dot{\varepsilon}_{d}$

where the plus sign corresponds to tension and the minus to compression. A generalisation of the elastic-viscoplastic constitutive model that includes strain dependent viscosity [23] is used by adopting the following relationship

$$
\eta\left(\varepsilon_{d}^{v}\right)= \begin{cases}\eta_{o}\left[1+s_{\infty}\left|\varepsilon_{d}^{v}\right|+\frac{s_{o}-s_{\infty}}{\alpha_{v}} \ln \left(\frac{1}{\left.1+e^{-\alpha_{v}\left(\left|\varepsilon_{d}^{v}\right|-\overline{-}_{v}\right.}\right)}\right)-\frac{s_{o}-s_{\infty}}{\alpha_{v}} \ln \left(\frac{1}{1+e^{\alpha_{v} \bar{\varepsilon}_{v}}}\right)\right] & \varepsilon_{d}^{v} \dot{\varepsilon}>0 \\ \eta_{o} & \varepsilon_{d}^{v} \dot{\varepsilon}<0\end{cases}
$$

where $\varepsilon_{d}^{v}$ is the strain of the viscous element of the model which can be calculated as follows

$$
\varepsilon_{d}^{v}=\varepsilon-\frac{\sigma-E_{p} \varepsilon_{d}^{v e}}{E_{v}}
$$

The dependence of viscosity on the viscous strain follows a bilinear curve when the viscous element is in tension and the strain rate is positive, or when the viscous element is in compression and the strain rate negative (corresponding to loading of the element either in tension or compression). In this region, the slope of the bilinear curve is $\eta_{o} s_{o}$ at low absolute viscous strains and $\eta_{o} s_{\infty}$ at high absolute viscous strains. Transition from one behaviour to the other occurs at absolute viscous strain $\bar{\varepsilon}_{v}$ with a breadth proportional to $1 / a_{v}$. In unloading, i.e. when strain rate and viscous strain have opposite signs, the viscosity has a constant value. A schematic of the typical behaviour produced by the shear element constitutive model in a loading-relaxation-reloading-unloading strain profile is shown in Fig. 2(b). The stress initially increases following the behaviour of a typical bias extension curve. During relaxation, it decays towards a low value. On reloading the stress increases rapidly and then follows the bias extension behaviour at high strains, whereas during unloading it decreases rapidly until it 
reaches the yield stress in compression. Then the material flows until strain becomes positive and the material starts to resist shear in the opposite direction.

It should be noted that the superposition assumption implied in Eqs. (5) and (9) is not strictly valid in large displacement analysis. A Green-Lagrange strain formulation is used in the model developed here. In this case the relative discrepancy when the deformation is distributed with weights $w$ and $1-w$ between the two strain components is given by the relationship

$D=w(1-w) \frac{2+2 \varepsilon_{t o t}-2 \sqrt{1+2 \varepsilon_{t o t}}}{\varepsilon_{t o t}}$

where $\varepsilon_{t o t}$ is the total strain and the weight $w$ ranges from 0 to 1 . The theoretical maximum of relative discrepancy occurs for maximum total strain and equal weights. The kinematics of the unit cell limits the Green-Lagrange strain of the diagonals to 0.5 , which corresponds to a relative error of $8 \%$. In practice, this error is lower because lock up of woven material limits the deformation to diagonal strains below 0.4. Furthermore, the part of the deformation driven by friction dominates the shear of woven material. This results in most of the deformation occurring as plastic strain. Similarly, for realistic process speeds the resistance of the viscous element is significantly lower than that of the elastic part of the Maxwell branch of the elasticviscoplastic model. Consequently, the weight attributed to plastic and viscous deformation is expected to be higher than $50 \%$ and the relative discrepancy significantly smaller than $8 \%$.

\subsection{Analysis of the unit cell response}

Fig. 3(a) shows the unit cell under a general three-dimensional state of stress. Forces $\mathbf{f}_{\mathbf{i}=1 . .4}$ at the four nodes result in stress normalised by the number of tows $\sigma_{\mathrm{i}=1 . .4}$ in tow elements and $\tau_{\mathbf{i}=1,2}$ in shear elements. The length and direction of tow elements is expressed by vector $\mathbf{r}_{\mathbf{i}=1.44}$. Analysis of the stress condition of the unit cell requires combination of force balance with compatibility conditions and constitutive relationships. A force balance at node 1 yields

$$
\frac{\mathbf{f}_{1}}{n_{t}}=\sigma_{4} \frac{\mathbf{r}_{4}}{\left\|\mathbf{r}_{4}\right\|}+\sigma_{1} \frac{\mathbf{r}_{1}}{\left\|\mathbf{r}_{1}\right\|}+\tau_{2} \frac{\mathbf{r}_{1}+\mathbf{r}_{2}}{\left\|\mathbf{r}_{1}+\mathbf{r}_{2}\right\|}
$$

where $n_{t}$ is the number of tows in the warp or weft direction in the unit cell, which can be calculated as $n_{t}=l_{t} / \Delta$, with $\Delta$ the initial unit cell size and $l_{t}$ the linear tow density in the warp/weft direction. 


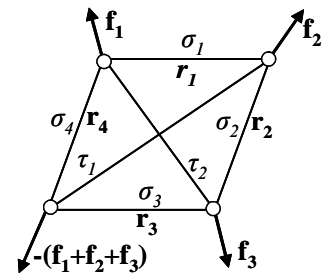

(a)

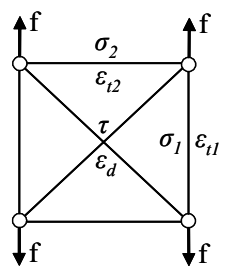

(b)

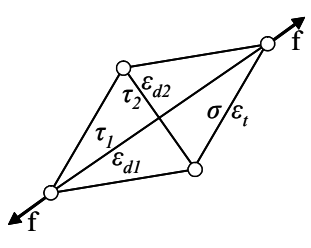

(c)

Fig. 3. State of stress of the unit cell of the simplified finite element model under (a) general loading, (b) tensile loading in the tow direction and (c) bias extension.

Three similar relationships can be obtained for the rest of the nodes, but only two of these are independent when the balance of all external forces is taken into account. The following compatibility condition must hold

$\sum_{i=1}^{4} \mathbf{r}_{\mathbf{i}}=0$

Constitutive relationships that relate stress to element length via a single valued mapping in the case of tow elements result in four expressions of the type

$\sigma_{i}=G\left(\frac{\left\|\mathbf{r}_{\mathbf{i}}\right\|^{2}-\Delta^{2}}{2 \Delta^{2}}\right)$

In the case of the elastic-viscoplastic shear elements the mapping involves a number of additional variables, expressed by a vector $\mathbf{q}$. Two expressions of the form

$\tau_{1}=H\left(\frac{\left\|\mathbf{r}_{2}+\mathbf{r}_{3}\right\|^{2}-\Delta^{2}}{2 \Delta^{2}}, \mathbf{q}\right)$

can be obtained from integration of the elastic-viscoplastic constitutive model described by Eqs. (5) to (9). Given the forces acting on the nodes, 6 element stresses and 4 element vectors that correspond to a total of 18 unknown variables characterise the state of stress. The number of available algebraic equations in the general case is also 18, 9 by the force balance, 3 by the compatibility condition and 6 by the constitutive relationships.

The solution of an equivalent system of equations is performed by the implicit finite element code used in the current implementation of the model (MSC.Marc). For the simple mechanical models used here, an explicit formation is not needed, considerably speeding up the solution time. However, it is useful to derive solutions in the cases of loading under tension and bias extension as these represent experimental conditions commonly used for obtaining material properties of woven textiles. Parameter identification for material submodels can then be performed efficiently using these rather than relying on finite element solutions. The two loading situations are depicted in Figs. 3(b) and 3(c). The symmetry of 
tensile loading results in equal force and strain in the two shear elements and equal responses of opposite tow elements of the unit cell. In bias extension, all tow elements of a unit cell under pure shear are under the same stress state. In a displacement controlled tension experiment the strain of tows aligned to the loading $\varepsilon_{t 1}$ is known. The strain of the other set of tows $\varepsilon_{t 2}$ and of shear elements $\varepsilon_{d}$ relate to the known strain as follows

$\varepsilon_{t 1}+\varepsilon_{t 2}=2 \varepsilon_{d}$

Combining Eq. (15) and the constitutive relationships (Eqs. (13) and (14)) with a force balance in the normal to the loading direction at one of the nodes yields

$H\left(\varepsilon_{d}, \mathbf{q}\right) \sqrt{\frac{1+4 \varepsilon_{d}-2 \varepsilon_{t 1}}{2+4 \varepsilon_{d}}} \varepsilon_{t 1}+G\left(2 \varepsilon_{d}-\varepsilon_{t 1}\right)=0$

This relationship can be used to calculate the strain of diagonal elements and combined with the corresponding constitutive model to estimate the shear elements force per tow $\tau$. Subsequently, nodal force can be calculated as follows

$f=\tau \sqrt{\frac{1+2 \varepsilon_{t 1}}{2+4 \varepsilon_{d}}} \varepsilon_{t 1}+G\left(\varepsilon_{t 1}\right)$

In bias extension tests the strain of the major diagonal is known from the experimental results and the compatibility condition becomes

$\varepsilon_{d 1}+\varepsilon_{d 2}=2 \varepsilon_{t}$

The strain of tow elements can be obtained by combining the compatibility condition with a force balance at one of the free nodes in the direction of the minor diagonal

$H\left(2 \varepsilon_{t}-\varepsilon_{d 1}, \mathbf{q}\right)+G\left(\varepsilon_{t}\right) \sqrt{\frac{1+4 \varepsilon_{t}-2 \varepsilon_{d 1}}{2+4 \varepsilon_{t}}}=0$

The normalised force of tow $\sigma$ and shear $\tau_{1}$ elements can be calculated from the constitutive model and the applied force is estimated as follows

$f=2 \sigma \sqrt{\frac{1+2 \varepsilon_{d 1}}{2+4 \varepsilon_{t}}} \varepsilon_{t 1}+\tau_{1}$

\section{Material properties}

\subsection{Material characterisation}

The information required for the development of appropriate constitutive models for the tow and diagonal shear elements of the unit cell was obtained using tensile and bias extension 
tests. The material was a $4 \times 4$ twill weave $3 \mathrm{k}$ carbon/1947 epoxy pre-preg manufactured by Hexcel. More details of the woven material are shown in Table 1.

\section{Table 1}

Properties of $3 k$ carbon/1947 epoxy $4 \times 4$ twill weave

\begin{tabular}{lll}
\hline Parameter & Value & Remarks \\
\hline Fibre areal weight & $280 \mathrm{~g} / \mathrm{m}^{2}$ & Quoted \\
Resin weight fraction & 0.42 & Quoted \\
Tow width & $1.3 \mathrm{~mm}$ & Measured \\
Linear tow density in warp/weft & 7.1 tows $/ \mathrm{cm}$ & Measured \\
direction & & \\
\hline
\end{tabular}

Tensile experiments were carried out on an Instron test machine. A gauge length of $10 \mathrm{~cm}$ was used and the deformation was measured using a laser extensometer. The load was applied using clamps of $2.5 \mathrm{~cm}$ width which was equal to the width of the samples. The speed of the experiments was $2 \mathrm{~mm} / \mathrm{min}$. Bias extension tests were carried out using the equipment described in [24]. A rectangular piece of woven material, $210 \mathrm{~mm} \times 70 \mathrm{~mm}$ in dimensions, was loaded with the warp and weft directions orientated initially at $\pm 45^{\circ}$ to the loading. The tests were performed at a constant relative velocity of the two ends of the specimen. Three velocities were used, 10, 40 and $160 \mathrm{~mm} / \mathrm{min}$. The applied force and the deformation of the diagonal of a square gauge region in the full shear part of the specimen shown in Fig. 4 were recorded. The initial size of the diagonal of the gauge region was $40 \mathrm{~mm}$.

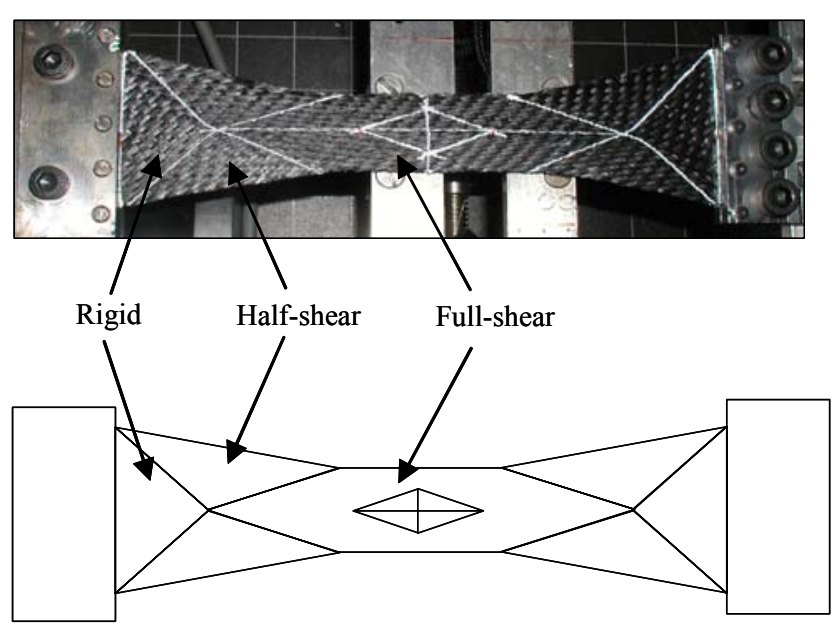

Fig. 4. Bias extension test setup and ideal pin-jointed kinematics. Deformation is measured along the diagonal of a central rhombic region. Initial dimensions of specimen: length $21 \mathrm{~cm}$, width $7 \mathrm{~cm}$. 
A constant and uniform strain rate of diagonal elements of the model in the full shear region is necessary for effective use of bias extension test results in the identification of the parameters of the elastic-viscoplastic material sub-model. The assumption of pin-jointed kinematics and the use of Green-Lagrange strain result in symmetry between the strains of the major and the minor diagonals of the gauge region, ensuring uniform absolute strain rate in the full-shear area. The Green-Lagrange strain of the diagonals $\varepsilon_{d}$ resulting from the application of constant crosshead speed $\dot{L}$ is given by the following relationship,

$\left|\varepsilon_{d}\right|=\frac{\dot{L} t}{L_{o}-W_{o}}+\frac{1}{2}\left(\frac{\dot{L} t}{L_{o}-W_{o}}\right)^{2}$

where $L_{o}$ is the initial length of the specimen, $W_{o}$ is the initial width of the specimen and $t$ denotes time. The strain evolution with time can be approximated by a linear relationship,

$\left|\bar{\varepsilon}_{d}\right|=\dot{\varepsilon}_{e f f} t$

where $\dot{\varepsilon}_{\text {eff }}$ is the effective constant strain rate, which can be calculated by minimising the integral $\int_{0}^{1 / 2}\left(\left|\bar{\varepsilon}_{d}\right|-\left|\varepsilon_{d}\right|\right)^{2} d \varepsilon_{d}$. The upper limit of the integral corresponds to the maximum GreenLagrange strain under the pin-jointed net assumption. The minimisation yields,

$\dot{\varepsilon}_{e f f}=\frac{\dot{L}}{L_{o}-W_{o}}\left[\frac{3 \sqrt{2}+5}{8}\right]$

The term $\dot{L} / L_{o}-W_{o}$ in Eq. (23) expresses the strain rate corresponding to engineering strain in the diagonal bar under tension. The effective Green-Lagrange strain rate is $15.5 \%$ higher than this value due to the last term in Eq. (23). The effective strain rates for the 10, 40 and 160 $\mathrm{mm} / \mathrm{min}$ experiments are $0.0013,0.0055$ and $0.022 \mathrm{~s}^{-1}$ respectively. Fig. 5 compares the evolution of experimental strains to strains calculated using Eqs. (22) and (23). It can be observed that the linear approximation follows experimental strains very closely and deviations are comparable to noise in the experimental data. The correlation between the experimental data and the approximation is 0.99 , indicating negligible errors. 


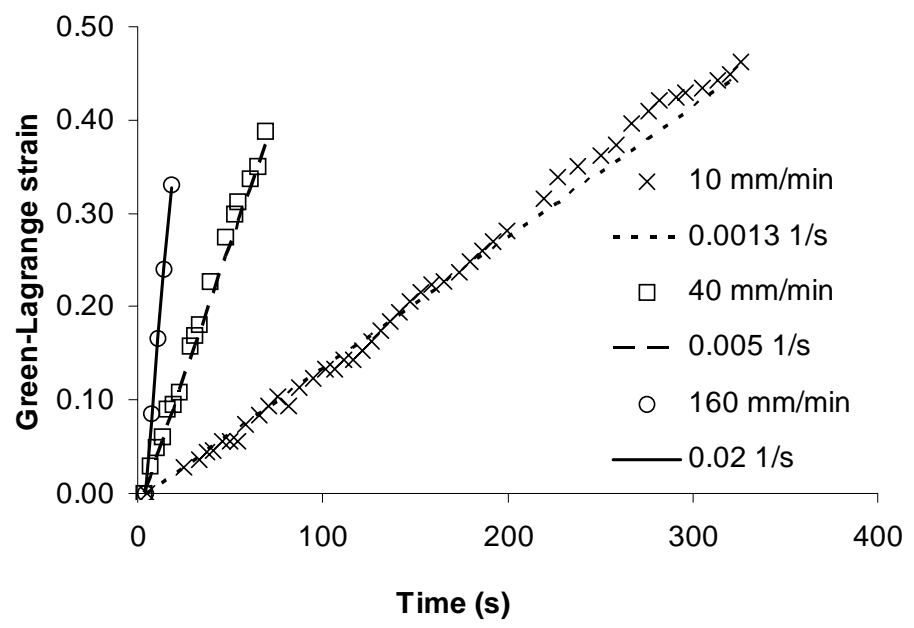

Fig. 5. Evolution of experimental strain and linear Green-Lagrange strain with time for the bias extension experiments and the correlation coefficients between experimental data and the predictions of Eqs. (22) and (23).

\subsection{Results and estimation of material sub-model parameters}

Fig. 6 illustrates the results of tensile and bias extension characterisation experiments. The typical bilinear behaviour caused by decrimping effects is observed in tension. Bias extension results show an initial force increase at low strains, followed by a low stiffness region and a large increase in stiffness at high strain. This behaviour is caused by the successive dominance of tow bending, friction at tow crossovers and lateral tow compression, as strain increases. Strong strain rate dependence of the response in bias extension is observed. Apparent shear compliance decreases with increasing rate, whereas the strain at which a transition from low to high shear stiffness behaviour increases. These observations are consistent with the viscous behaviour of a thermosetting matrix. Measured force was normalised by the number of tows of the corresponding specimen (17.8 tows in the tensile test and 35.3 in the bias extension test). Eqs. (15) to (17) were used to simulate tension and Eqs. (18) to (20) bias extension in an incremental scheme. Non-linear equations such as Eqs. (16) and (19) were solved using the Newton-Raphson method. Inversion of the model response in order to estimate the parameters of the constitutive relationships was performed using the Generalised Reduced Gradient Nonlinear Optimisation method implemented in Microsoft Excel [25]. The objective of inversion was the minimisation of the average absolute difference of force predicted by the model and measured during the experiments. The nonlinear character of the problem may create difficulties in the fitting procedure due to the existence of numerous local minima that induce a strong dependence of the solution on the 
initial guess. However, the clear physical interpretation of model parameters allows the selection of a high quality initial solution vector that results in a robust fit.

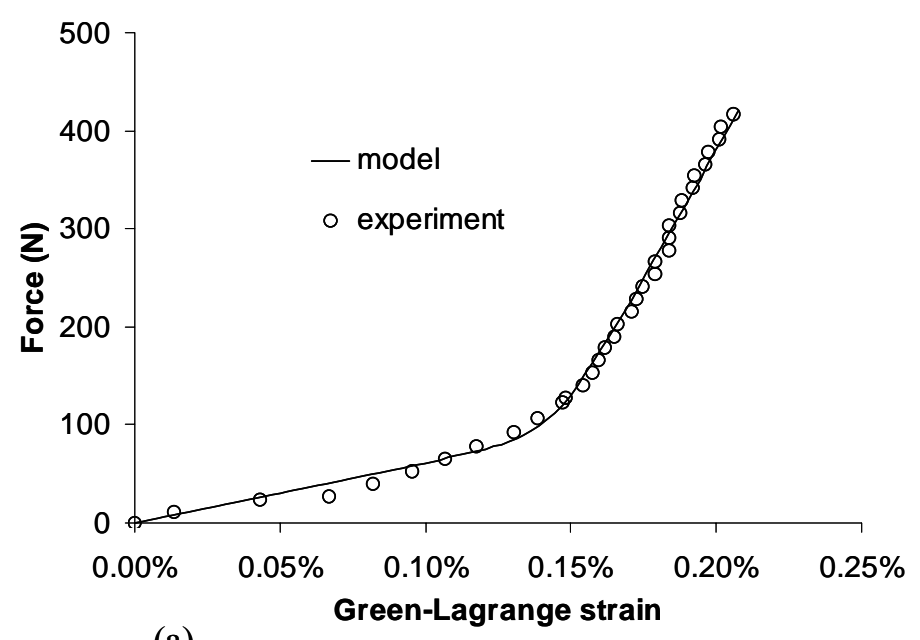

(a)

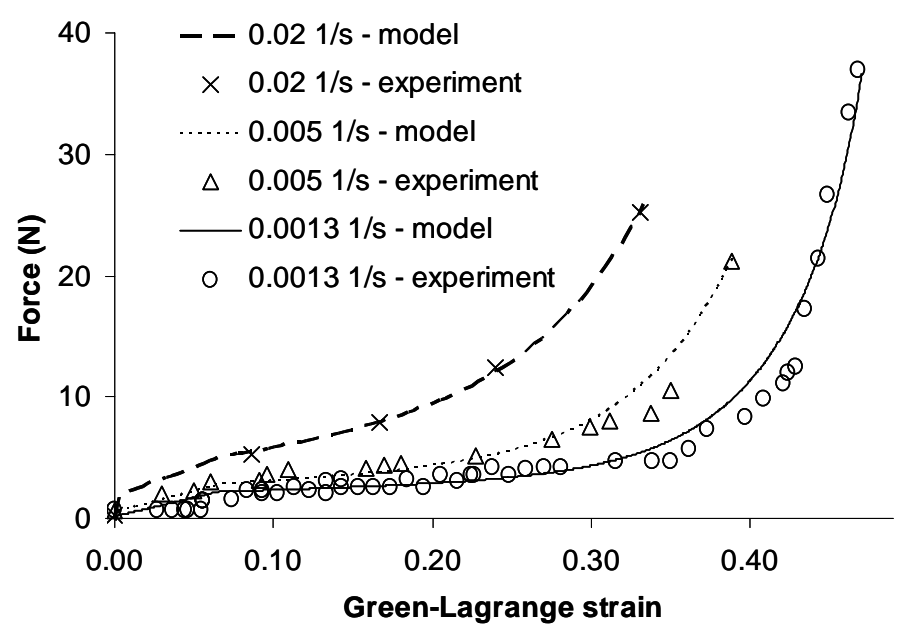

(b)

Fig. 6. Comparison between experimental and model results in (a) tension and (b) bias extension for $4 \times 4$ twill weave $3 k$ carbon $/ 1947$ pre-preg.

Parameter $\sigma_{o}$ corresponds to the stress level at which the first increase in stress governed by tow bending in bias extension tests stops. Parameter $\eta_{o}$ expresses the amplitude of viscosity and can be inferred by the dependence of stress on strain rate in bias extension tests at low to intermediate strains. Parameters $s_{o}$ and $s_{\infty}$ can be inferred from the low and high strain slopes of the low strain rate bias extension curve once an initial guess for $\eta_{o}$ is set. Similarly, initial guesses for $\alpha_{v}$ and $\bar{\varepsilon}_{v}$ can be obtained by the transition strain and the breadth of transition of the low strain rate bias extension curve. The two moduli of the elasticviscoplastic model ( $E_{p}$ and $E_{v}$ ) are pre-estimated by the initial slope of the bias extension curves. The limit moduli of the tow elements sub-model ( $E_{o}$ and $\left.E_{\infty}\right)$ can be inferred with 
high accuracy using the initial and final slopes of the tensile curve. Similarly, initial guesses for the breadth parameter and the strain at the transition from low to high modulus ( $\alpha$ and $\bar{\varepsilon}$ ) are obtained from the tensile test results.

Table 2

\section{Parameters of the constitutive models and model fitting error and sensitivity of response and error to model parameters}

\begin{tabular}{llllll}
\hline Parameter & Value & $\begin{array}{l}\text { Tensile } \\
\text { sensitivity }\end{array}$ & $\begin{array}{l}\text { error } \\
\text { error sensitivity }\end{array}$ & $\begin{array}{l}\text { extensionsile force } \\
\text { sensitivity }\end{array}$ & $\begin{array}{l}\text { Bias extension force } \\
\text { sensitivity }\end{array}$ \\
\hline$\sigma_{o}$ & $2.6210^{-2} \mathrm{~N} /$ tow & 0.0 & $6.310^{-1}$ & 0.0 & $2.910^{-1}$ \\
$\eta_{o}$ & $1.37 \mathrm{~N} /$ tow & $1.110^{-5}$ & 2.8 & $1.210^{-5}$ & $5.510^{-1}$ \\
$S_{o}$ & 7.48 & $-1.010^{-8}$ & $6.410^{-1}$ & $4.410^{-8}$ & $1.610^{-1}$ \\
$s_{\infty}$ & $5.3910^{3}$ & $-4.510^{-10}$ & 1.8 & $1.910^{-9}$ & $2.510^{-1}$ \\
$\alpha_{v}$ & $1.9010^{1}$ & $3.910^{-9}$ & 5.0 & $-1.610^{-8}$ & -1.1 \\
$\bar{\varepsilon}_{v}$ & $5.1210^{-1}$ & $3.910^{-9}$ & $1.110^{1}$ & $-1.610^{-8}$ & -2.2 \\
$E_{v}$ & $7.89 \mathrm{~N} /$ tow & $2.110^{-9}$ & $9.610^{-1}$ & $1.510^{-7}$ & $1.210^{-1}$ \\
$E_{p}$ & $3.9010^{-1} \mathrm{~N} /$ tow & $-4.010^{-6}$ & $1.310^{-1}$ & $2.310^{-5}$ & $2.710^{-2}$ \\
$E_{o}$ & $3.4110^{3} \mathrm{~N} /$ tow & 1.2 & $-3.810^{-3}$ & $5.110^{-1}$ & $7.310^{-4}$ \\
$E_{\infty}$ & $2.9110^{4} \mathrm{~N} /$ tow & 1.1 & $-3.210^{-10}$ & $4.810^{-1}$ & $4.310^{-11}$ \\
$\bar{\varepsilon}$ & $1.4410^{-3}$ & $2.910^{1}$ & $4.310^{-9}$ & -1.8 & $-5.810^{-10}$ \\
$\alpha$ & $1.3310^{4}$ & $5.310^{-3}$ & $4.110^{-9}$ & $-3.510^{-2}$ & $-5.710^{-10}$ \\
\hline
\end{tabular}

The results of model fitting are summarised in Table 2. The average error is $7.1 \mathrm{~N}$ in tensile force estimation and $0.6 \mathrm{~N}$ in bias extension. The corresponding relative errors are in the range of $7-9 \%$. The quality of the fit is illustrated in Fig. 6 . The tensile curve is followed by the model. The general form of the bias extension simulation results matches the experiments successfully and strain rate dependence and the transition from tow crossover friction to tow compression are followed closely. The sensitivity of the model fit and of its response to the estimated parameters was investigated by calculating sensitivity coefficients of the fitting error and the average force over all experiments. To calculate the sensitivity coefficients, each independent parameter was increased by $2.5 \%$ and the percentage variation in the model response and fitting error was calculated and divided by the percentage change in the parameter. Investigation of sensitivity at higher variation levels indicated that the linearity assumption underlying this analysis is valid for changes up to $5 \%$. The results are summarised in Table 2. It is found that the tensile error and response are sensitive only to the parameters of the tow element material sub-model. Similarly the bias extension error and response are 
sensitive only to the parameters of the shear element material sub-model. Consequently, the parameter estimation problem can be solved separately for the two sub-models and experiments. However, the general formulation used here may be necessary in other woven materials, e.g. when decrimping modulus or fibre modulus is low. The bias extension fitting error shows high sensitivity to the viscous transition strain, the amplitude of viscosity and the breadth parameter of the viscosity transition. The tension fitting error is most sensitive to the transition strain. The model response is very sensitive to both transition strain and the viscosity amplitude. The two moduli of the elastic-viscoplastic model have low influence on both the error and the model response because the experimental data do not include results of relaxation or unloading tests that would manifest high sensitivities to these parameters.

\section{Modelling of hemisphere forming and validation}

The simplified forming model described in the previous sections was implemented in MSC.Marc to model the forming of a hemisphere, which was also investigated experimentally for validation purposes. The truss was simulated using an assembly of two-node bar elements. Appropriate user defined subroutines were developed for the incorporation of the viscoplastic stress-strain response of shear elements, of wrinkling and non-linear elastic behaviour of tow elements and of the perfectly plastic behaviour of friction elements. Material properties produced by experimental analysis and parameter identification are normalised with respect to the number of tows. Consequently, properties of the elements of the unit cell need to be scaled by multiplying by $n_{t} / A_{e}$, where $n_{t}$ is the number of warp/weft tows and $A_{e}$ the crosssectional area of the bar element. Tooling was modelled as a group of rigid surfaces and contact between the woven material and tooling was included. The symmetry of the hemisphere combined with the bi-directional properties of the material allows simulations to be based on a one quarter model.

\subsection{Hemisphere forming experiments}

Hemisphere forming experiments were carried out using a stamp forming process with two matched tools set on a hydraulic press. Draw-in of the pre-preg was controlled using a blankholder comprising two square clamping plates with central circular openings. The nominal radius of the hemispherical part of the tool was $60 \mathrm{~mm}$, while the hemisphere was connected with the tool base via a fillet of $20 \mathrm{~mm}$ in radius. The diameter of the openings of the clamping plates was $170 \mathrm{~mm}$, allowing movement of one of the hemispherical tools. The 
force acting on the clamping plates was controlled using four springs which were set at appropriate compression by tightening a bolt. Pre-impregnated woven material was placed on the blank-holder which was then mounted in place. Release agent was applied to all tool surfaces prior to the placement of the material to allow easy demoulding after curing. Experiments were conducted using the $4 \times 4$ twill weave $3 \mathrm{k}$ carbon/1947 epoxy prepreg (Hexcel) used in material characterisation. The size of the sheet of pre-impregnated sheet was $240 \times 240 \mathrm{~mm}$. Prior to experiments white lines were drawn on the woven material following the direction of tows to form a grid, $10 \mathrm{~mm}$ in size. Forming was performed at room temperature using a total holding force of $133 \mathrm{~N}$, which included contributions from the four springs and the weight of the top clamping plate. The travelling distance of the movable tool was $80 \mathrm{~mm}$, the forming speed was approximately $2 \mathrm{~mm} / \mathrm{s}$ and the duration of forming $40 \mathrm{~s}$. After completion of forming, the assembly was heated up to $120{ }^{\circ} \mathrm{C}$ and was kept at that temperature for an hour to cure the epoxy resin. The final glass transition temperature of the cured material is $143{ }^{\circ} \mathrm{C}$.

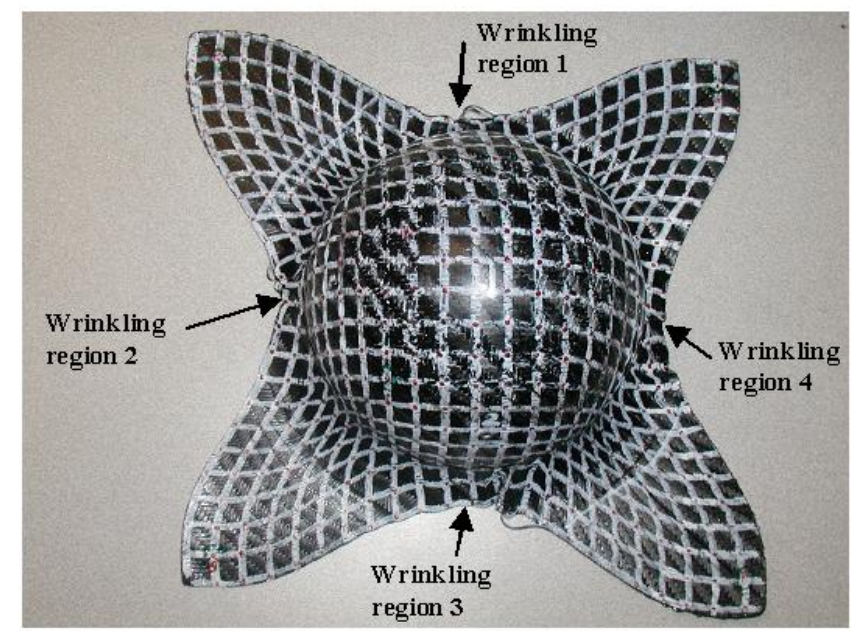

(a)

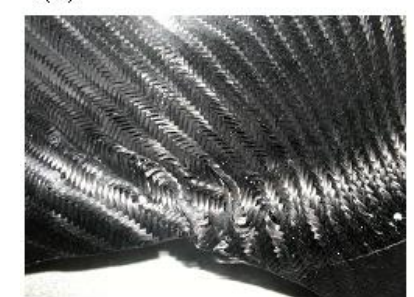

(b)

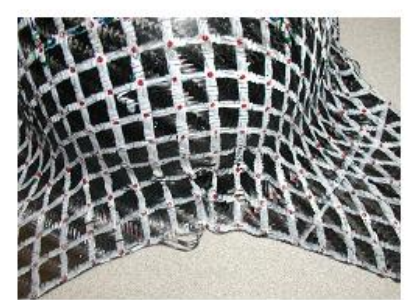

(c)

Fig. 7. Forming of a $4 \times 4$ twill weave $3 k$ carbon $/ 1947$ hemisphere. (a) Formed specimen. (b) Detail of the occurrence of wrinkling in region 1 in the internal surface of the specimen. (c) Detail of the occurrence of wrinkling in region 1 in the external surface of the specimen. The spacing of the grid is $10 \mathrm{~mm}$. 
Fig. 7 shows the cured specimen obtained in the experiment. It can be observed that high shear occurs at diagonal positions along the bias direction of the woven material while the material on the apex of the hemisphere is undeformed. Wrinkling due to tow buckling occurs at four positions along the direction of the two sets of tows in the area of the fillet. The direction of buckling is parallel to the sides of the specimen. Excessive compression can result in folding of the material as it can be seen in Fig. 7(c).

The overall shape of the formed specimen is slightly asymmetric. The way wrinkling strain is distributed in the areas of concentrated tow buckling and is also asymmetric. In the region shown in Fig. 7(c) and its diametrically opposite side, wrinkling results in folding. In contrast, wrinkling at the other two areas of concentrated buckling is distributed over an area covering a few squares of the grid. The observed asymmetry can be attributed to initial variations in the angle between tows and variations in the application of holding force from the blank holder. The positions of the nodes of the grid, shown as dots on the formed specimen in Fig. 7, were measured using an Omicron 3-D coordinate measurement system. The specimen was set on a table, the measuring head of the device was moved to each of the nodes and its position in space was recorded. In order to remove the effects of asymmetry and provide a reliable set of deformation measurements for comparison with the forming model, an average of the grid positions on the four quarters of the hemisphere was calculated. The point cloud was post processed to calculate the

strains and shear angle of the unit cells of the grid.

\subsection{Model convergence and validation}

The spatial convergence of the model was investigated by simulating hemisphere forming using meshes of different sizes. One quarter of the $240 \times 240 \mathrm{~mm}$ sheet of woven material was represented using unit cell sizes in the range from 60 to $8.6 \mathrm{~mm}$, resulting in models with number of nodes ranging from 9 to 225. Solution times using MSC.Marc on a Pentium 42.8 $\mathrm{GHz}$ PC were in the order of $5 \mathrm{~s}$ for the 9 node model and in the order of $60 \mathrm{~s}$ for the 225 node model. These times are significantly shorter than solution times for shell based comprehensive finite element simulations of forming, which are expected to be in the order of hours, and significantly longer than execution times for kinematic models, which are in the order of seconds. The total clamping force for these model runs was $133 \mathrm{~N}$.

Fig. 8 illustrates the draped pattern predicted by the model at various unit cell sizes. The geometry of the tool surface is followed closely for unit cell sizes smaller than $15 \mathrm{~mm}$ (81 nodes in the finite element representation of a quarter of the woven sheet) which corresponds 
to a quarter of the hemisphere radius. The geometric fit is poor for mesh densities lower than this. The theoretical formed pattern shows maximum shear at diagonal positions aligned to the bias direction of the woven textile, which is qualitatively similar to the pattern seen in the experimental observations. The dotted lines in Fig. 8 indicate regions where significant wrinkling is evident. This occurs at normal positions aligned to the direction of warp and weft tows on the base of the hemisphere, whereas compression due to wrinkling follows the direction of the corresponding side of the woven material sheet.

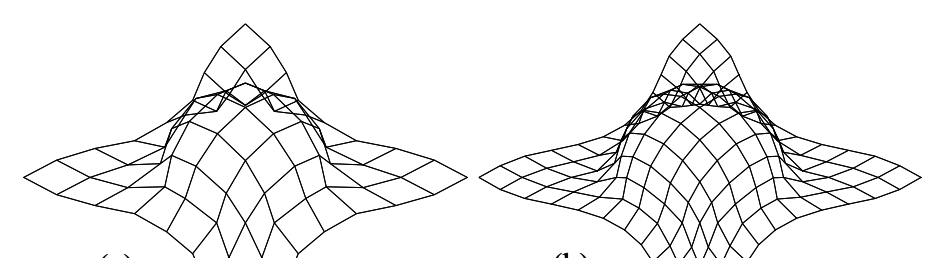

(a)

(b)

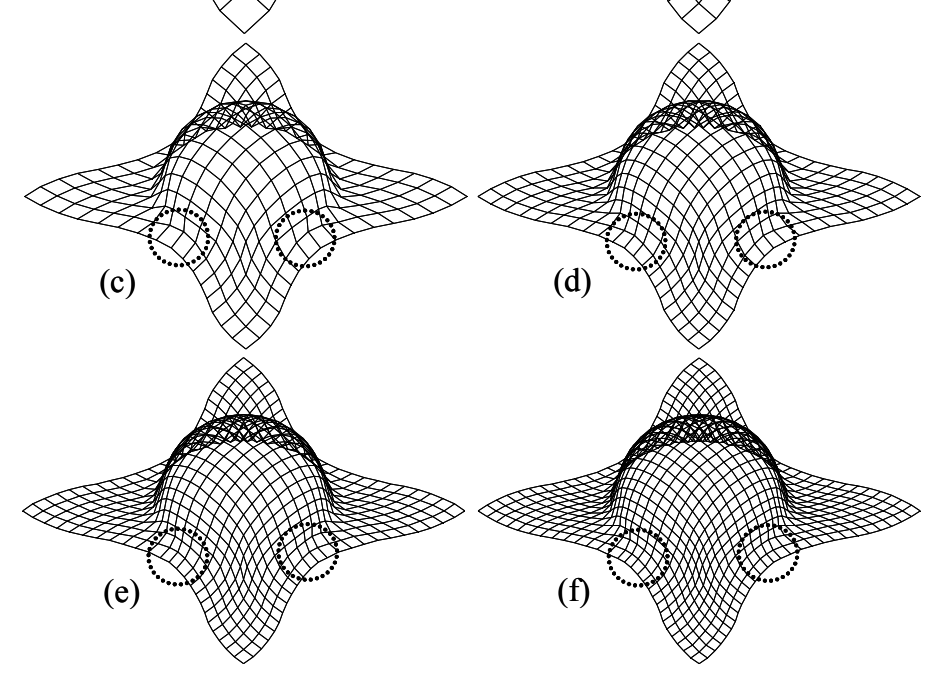

Fig. 8. Formed patterns at different unit cell sizes. One quarter of the hemisphere is modelled using (a) 25, (b) 49, (c) 81, (d) 121, (e) 169 and (f) 225 nodes. The dotted circles indicate regions of concentrated buckling.

Fig. 9 illustrates the dependence of maximum shear angle, total wrinkling strain and average error in total node displacement as a function of the number of nodes of the forming model. Total wrinkling strain was calculated by summing up wrinkling deformation over all elements with negative strains and dividing this by the total length of tow elements of the model. 


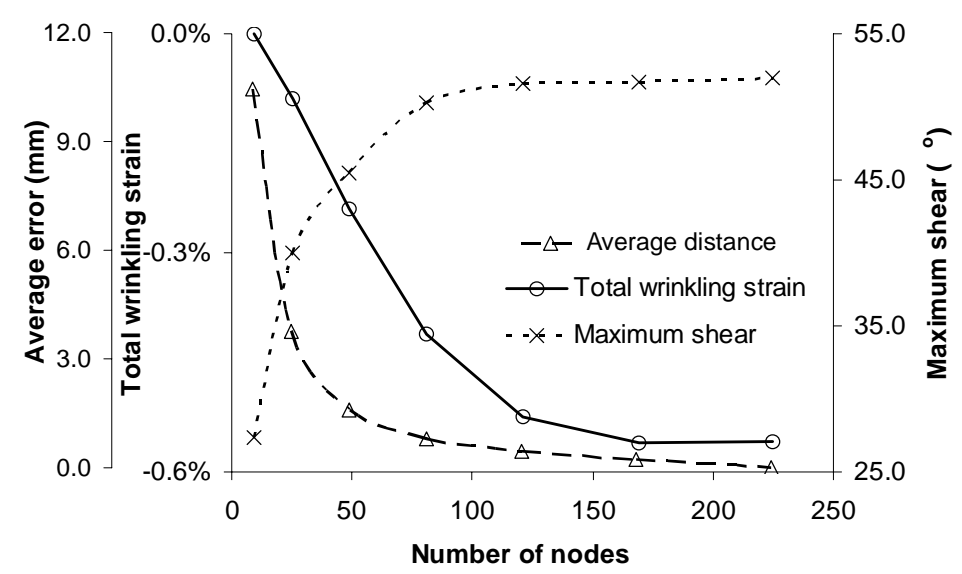

Fig. 9. Dependence of total wrinkling strain, maximum shear and average nodal distance

from the results of the finest model on the total number of nodes of the quarter model.

The average error in total displacement was estimated as the difference between the predicted final nodal position for each model and the corresponding position predicted by the finest model, taking an average over 169 points of a $13 \times 13$ square grid on the sheet of the woven material. The error drops steeply and becomes negligible with models having over 100 nodes or for unit cell sizes lower than $12 \mathrm{~mm}$. At a $10 \mathrm{~mm}$ unit cell size, the average error in displacement becomes approximately $0.25 \mathrm{~mm}$, or $2.5 \%$ of the unit cell size. The estimated maximum shear angle increases with increasing number of nodes as a result of the improved geometric fit with decreasing unit cell size. The value reaches a plateau beyond 100 nodes. Total wrinkling strain shows sensitivity to the mesh size up to 169 nodes or $10 \mathrm{~mm}$ unit cell size. Initially, predicted total wrinkling strain drops as the unit cell size decreases. For unit cell sizes below $10 \mathrm{~mm}$ the prediction for total wrinkling strain stabilises to a value of about $0.55 \%$. Overall, model predictions show stability with respect to mesh refinement. Nodal displacement and shear values converge for unit cell sizes below $12 \mathrm{~mm}$, whereas a unit cell size of $10 \mathrm{~mm}$ is required to reach a stable value of total wrinkling strain. Consequently, a unit cell of $10 \mathrm{~mm}$ is appropriate for modelling of the hemisphere geometry. It should be noted that this value is one sixth of the hemisphere radius and half the radius of the base fillet. Convergence of the model for relatively coarse meshes such as those represented in Figs 9(e) and $9(\mathrm{f})$ originates in the simplified representation of wrinkling effects and is one of the reasons for its computational efficiency. 


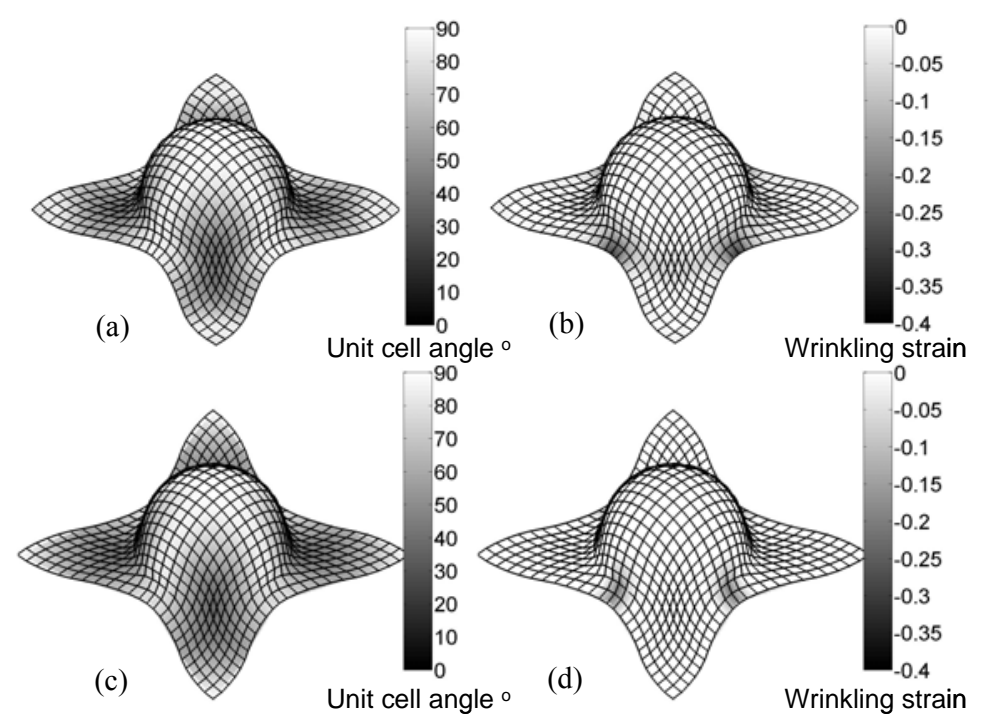

Fig. 10. Comparison of experimental measurements: (a) unit cell angle (b) Green-Lagrange wrinkling strain distribution, with model predictions: (c) unit cell angle (d) Green-Lagrange wrinkling strain distribution.

Fig. 10 illustrates the formed patterns observed in experiments and predicted by the model using a unit cell size of $10 \mathrm{~mm}$, along with the shear and wrinkling strain distributions. Model results for the shear distributions follow experimental results very closely, with high shear at four locations aligned to the bias direction and negligible shear on the apex. The shape of the formed sheet predicted by the model is very close to that observed in experimental forming, with the only difference being a slightly higher extension in the diagonal direction. The model locates correctly regions of concentrated wrinkling due to tow buckling at four normal positions aligned to the tow directions along the base of the hemisphere. The maximum shear measured in the experiment is $50.2^{\circ}$, whereas the corresponding model value is $51.4^{\circ}$. The minimum value of Green-Lagrange wrinkling strain is 0.31 and 0.21 in the experiment and the model predictions, respectively. The average forming prediction error, calculated as the average distance between the final positions of points of the square grid measured and predicted by the model, is $1.57 \mathrm{~mm}$, which is about $4 \%$ of the average distance between final and initial positions of the nodes of the grid. 


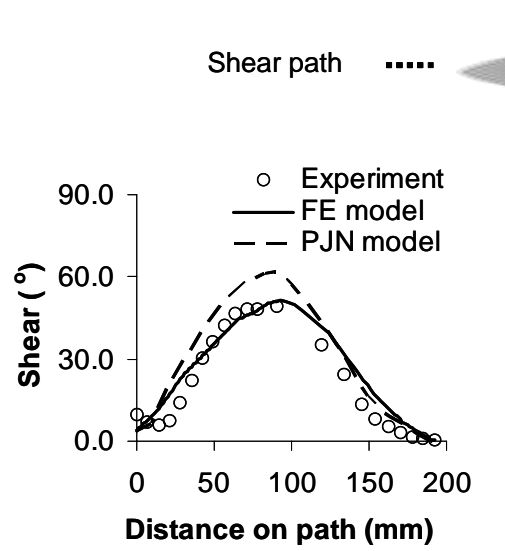

(a)

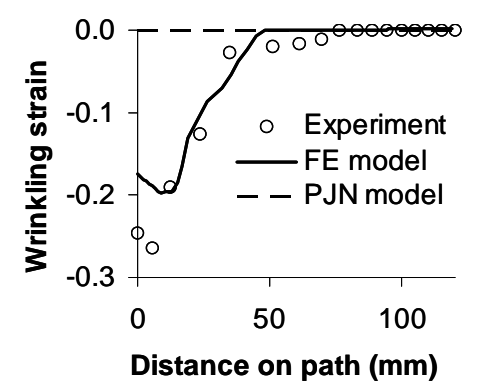

(b)

Fig. 11. Comparison of experimental results, finite element (FE) and kinematic (PJN) model predictions. (a) Shear angle along a diagonal path and (b) Green-Lagrange wrinkling strain along a normal path.

Fig. 11(a) provides a quantitative comparison of the shear distribution along a path of high shear starting at one of the corners of the woven material sheet and ending on the apex of the hemisphere. The experimental results indicate very low shear at the corner which increases to a maximum in the middle of the path and a subsequent drop towards zero as the path approaches the apex of the hemisphere. The model reproduces the general behaviour closely with small discrepancies in the corner region where the model shows a monotonic increase of shear, whereas the experiment indicates a slight decrease in shear in a narrow region before the increase. The results of a kinematic simulation are also shown in Fig. 11(a). The kinematic model reproduces the experimental behaviour of very low shear in the corner, negligible shear on the apex and maximum shear in the middle of the diagonal path. However, maximum shear predicted by the kinematic simulation is significantly higher, reaching values of $61^{\circ}$, due to the assumed inextensibility and incompressibility of tows. Fig. 11(b) shows a similar comparison for Green-Lagrange wrinkling strain along a path starting in the middle of a side of the base and ending on the apex. Measured wrinkling reaches its absolute maximum very near the base and then decreases until it stabilises to small absolute values at a distance of about $35 \mathrm{~mm}$. Measured wrinkling becomes negligible at about $45 \mathrm{~mm}$ from the apex. Model results start from a lower absolute Green-Lagrange wrinkling strain and reproduce the initial absolute wrinkling strain increase over the short distance observed in the experiment. Then wrinkling decreases over a region of about $40 \mathrm{~mm}$ and becomes negligible at about $70 \mathrm{~mm}$ from the apex.

Overall, the mode reproduces successfully the shear distribution over the formed component. Furthermore, regions of the formed hemisphere where wrinkling due to tow buckling occurs 
are identified correctly, while maximum absolute wrinkling is underestimated by the model. It should be noted that shear information obtained from the model can also be used for the identification of areas where wrinkling due to shear lock-up occurs, based on the expected one-to-one correspondence between shear strain and wrinkling strain of this type.

\subsection{Sensitivity study}

The proposed forming model can be used to investigate the effect of process variables on forming. Here the sensitivity of model response to the forming speed and holding force is explored to demonstrate the applicability of the rate dependent model in process optimisation. A unit cell size of $10 \mathrm{~mm}$ was used, as indicated by the mesh convergence study presented in the previous section. In the investigation of the speed effect, hemisphere forming of the $4 \times 4$ twill carbon/epoxy pre-preg was simulated for forming durations in the range of 8 to $240 \mathrm{~s}$. This corresponds to forming speeds in the range of 0.33 to $10 \mathrm{~mm} / \mathrm{s}$. The total holding force was kept constant at $133 \mathrm{~N}$. For the investigation of the effect of holding force, the forming duration was kept constant at $40 \mathrm{~s}$, i.e. a forming speed of $2 \mathrm{~mm} / \mathrm{s}$, and the total holding force was varied in the range of 70 to $800 \mathrm{~N}$.

The results of the forming model for the sensitivity to speed are illustrated in Fig. 12(a). The absolute value of total wrinkling decreases with decreasing forming speed as a result of the increase in shear compliance of the unit cell with a fall in strain rate. The maximum shear angle decreases with speed due to strain rate effects. The results of forming are very sensitive to speed at high rates, whereas sensitivity is minimum at very low speeds with both the total wrinkling and maximum shear reaching plateau values for forming durations higher than 100 s. The decrease in absolute total wrinkling strain from $1.1 \%$ at high speeds to $0.3 \%$ at low speeds is significant. The corresponding variation in maximum shear is relatively low, in the range of $3^{\circ}$. These results indicate that absolute total wrinkling and maximum shear are negatively correlated. This is a consequence of the fact that the mechanisms of wrinkling due to tow buckling and shear are competitive modes of deformation of the woven textile as it conforms on the doubly curved tool. Thus, conditions that promote shear, such as slow forming of a rate dependent textile, result in less wrinkling and vice versa. The extreme cases representing dominance of one of these mechanisms are the pin-jointed net in which resistance to shear is negligible and deformation occurs exclusively by shear, and a sheet of isotropic material in which resistance to shear is high and deformation during forming occurs mainly by wrinkling. 


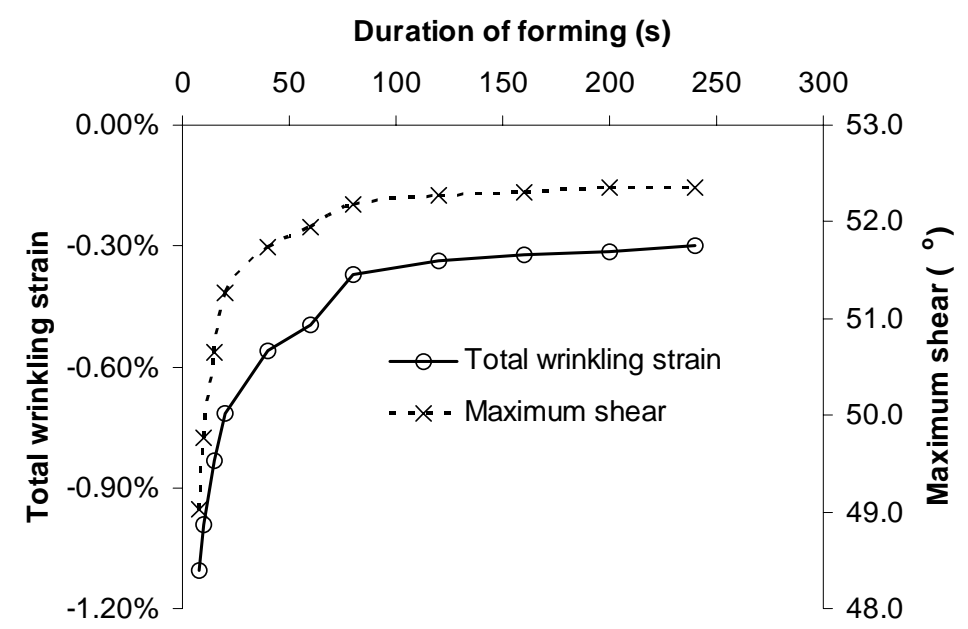

(a)

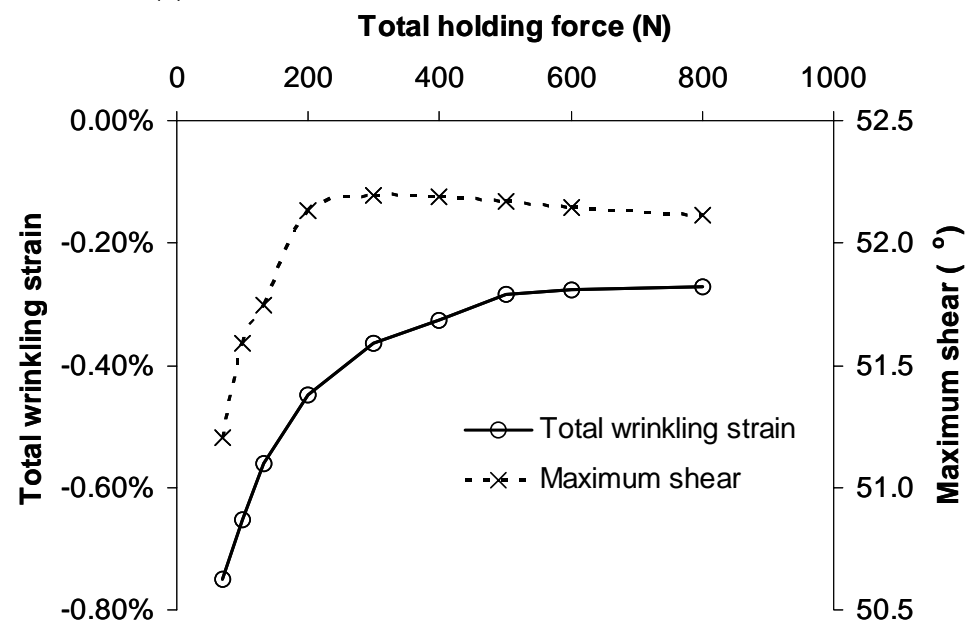

(b)

Fig. 12. Effect of (a) forming speed and (b) total holding force on the maximum shear angle and the wrinkling in the forming of the hemisphere. The unit cell size is $10 \mathrm{~mm}$. Total wrinkling strain is calculated as the ratio of the sum of wrinkling deformation to the total tow length of the model.

The effect of increasing the holding force, as illustrated in Fig. 12(b), is similar to that observed with decreasing forming speed. Absolute wrinkling decreases from $0.7 \%$ at $70 \mathrm{~N}$ to $0.25 \%$ at $800 \mathrm{~N}$ because of an increase in membrane forces that constrain compression of tows. The sensitivity of total wrinkling to blank holder force is high at low holding forces and decreases with increasing force, reaching an almost constant value for holding forces over 500 N. Maximum shear increases with force for low force values. For total holding forces over 200 N, maximum shear shows a slowly decreasing trend with force. This result is counterintuitive, as an increase in total holding force is expected to result in higher membrane force in the bias direction inducing higher shear in the same woven material under identical 
conditions. This behaviour, which is manifested at low forces, changes when membrane forces are high and can induce significant decrimping. Tow extension results in deformation in the tow direction, which contributes to deformation by elongation instead of shear. For example, in the case of total holding force of $800 \mathrm{~N}$, tow strain reaches values close to $0.5 \%$.

\section{Conclusions}

A simplified finite element forming model for pre-impregnated woven textiles is proposed. This simulates the material as a truss of tow and shear elements and can capture wrinkling due to buckling of tows and strain rate dependent shear. The unit cell of the model comprises a network of pin-jointed bars. Stiff elements represent the tows of the woven material while wrinkling is incorporated in the model by deactivating tow elements that undergo compression. The behaviour of the tow elements is non-linear elastic with suitable material properties extracted from tensile tests. Non-linear elastic-viscoplastic elements in the diagonals of the unit cell introduce the rate dependence in shear of the pre-impregnated woven material. A phenomenological elastic-viscoplastic constitutive model is developed, based on the results of bias extension tests at different strain rates. Friction with the tool surface is incorporated by a group of perfectly plastic elements that exert a constant force at nodes of the woven material in contact with the blank holder. The model includes the main mechanical effects, while retaining a simplicity which allows effective material parameter identification and iterative execution within an optimisation scheme.

The model is tested against experimental results obtained in the forming of a woven carbon/epoxy hemisphere. A mesh convergence study shows that the results of the model are stable with mesh refinement. A unit cell with a size 6 times smaller than the radius of the hemisphere ensures convergence of the model. The results of the comparison with experimental measurements suggest that the model reproduces successfully the shear field and the occurrence of wrinkling due to buckling. Maximum shear values are reproduced successfully and regions of significant wrinkling are identified correctly, while maximum wrinkling is underestimated.

The proposed model can be implemented easily within a non-linear finite element code that has an appropriate large displacement formulation, implements rate dependent material properties and allows element activation/deactivation. The results of a sensitivity study show that the forming process can be influenced significantly by forming speed and holding force. The simple character of the model allows a computationally efficient incorporation of strain 
rate dependence, wrinkling and influence of peripheral force in a forming simulation. Combination of the simplified simulation with optimisation tools can lead to efficient process design. Future work on process design for industrial components with complicated geometries will use this simplified and computationally efficient method as its core.

\section{Acknowledgements}

Contributions from the University of Nottingham, EPSRC, CONACyT, MSC.Software Ltd, DSTL, UK Ministry of Defence, Hexcel Composites, Granta Design Ltd., Ford Motor Company Ltd., Polynorm Plastics (UK) Ltd., Virtual Prototyping Solutions Ltd., SaintGobain Vetrotex International SA and ESI Software SA are gratefully acknowledged. The authors are grateful to Dr Phillip Harrison and the University of Nottingham for their assistance with the forming experiments.

\section{References}

1. Van Der Weeen F. Algorithms for draping fabrics on doubly curved surfaces. Int J Numer Meth Eng 1991;31(7):1415-1426.

2. Rudd CD, Rice EV, Bulmer LJ, Long AC. Process modeling and design for resin transfer molding. Plast Rubber Compos Process Applic 1993;20(2):67-76

3. Trochu F, Hammami A, Benoit Y. Prediction of fibre orientation and net shape definition of complex composite parts. Compos Part A-Appl S 1996;27(4):319-328.

4. Potluri P, Sharma S, Ramgulan R. Comprehensive drape modelling for moulding 3D textile preforms. Compos Part A-Appl S 2001;32(10):1415-1424.

5. Picket AK, Queckborner T,De Luca P, Haug E. An explicit finite element solution for the forming prediction of continuous fibre-reinforced thermoplastic sheets. Compos Manuf 1995;6(3-4):237-243.

6. Dong L, Lekakou C, Bader MG. Solid-mechanics finite element simulations of the draping of fabrics: a sensitivity analysis. Compos Part A-Appl S 2000;31(7):639-652.

7. Dong L, Lekakou C, Bader MG. Processing of composites: Simulation of the draping of fabrics with updated material behaviour law. J Compos Mater 2001;35(2):138-163.

8. Cao J, Xue P, Peng X, Krishnan N. An approach in modelling the temperature effect in thermo-stamping of woven composites. Compos Struct 2003;61(4):413-420. 
9. Sidhu RMJS, Averill RC, Riaz M, Pourboghrat F. Finite element analysis of textile composite preform stamping. Compos Struct 2001;52(3-4):483-497.

10. Cherouat A, Billoet JL. Mechanical and numerical modelling of composite manufacturing processes deep-drawing and laying-up of thin pre-impregnated woven fabrics. J Mater Process Tech 2001;118(1-3):460-471.

11. Sharma SB, Sutcliffe MPF.. A simplified finite element model for draping of woven material. Compos Part A-Appl S 2004;35(6):637-643.

12. McGuinness GB, Bradaigh CM. Development of rheological models for forming flows and picture-frame shear testing of fabric reinforced thermoplastic sheets. J Non-Newton Fluid Mech 1997;73(1-2):1-28.

13. Spencer AJM. Theory of fabric-reinforced viscous fluids. Compos Part A-Appl S 2000;31(12):1311-1321.

14. Spencer AJM. A theory of viscoplasticity for fabric-reinforced composites. J Mech Phys Solids 2001;49(11):2667-2687.

15. Peng X, Cao J. A dual homogenization and finite element approach for material characterization of textile composites. Compos Part B-Eng 2002;33(1):45-56.

16. Harrison P, Clifford MJ, Long AC, Rudd CD. A constituent-based predictive approach to modelling the rheology of viscous textile composites. Compos Part A-Appl S 2005;34(78):915-931.

17 Prodromou AG, Chen J. On the relationship between shear angle and wrinkling of textile composite preforms. Compos Part A-Appl S 2007;28(5):491-503.

18. Miller RK, HedgePeth JM, Weingarten VI, Das P, Kahyai S, Finite element analysis of partly wrinkled membranes. Comput Struct 1985;20(1-3):631-639.

19. Boisse P, Zouari B, Gasser A. A mesoscopic approach for the simulation of woven fibre composite forming. Compos S Techol 2005;65(3-4):429-436.

20. Potluri P, Parlak I, Ramgulam R, Sagar TV. Analysis of tow deformations in textile preforms subjected to forming forces. Compos S Techol 2006;66(2):297-305.

21. Liu L, Chen J, Gorczyza JL, Sherwood JA. Modelling of friction and shear in thermostamping of composites - Part II. J Compos Mater 2004;38(21):1931-1947.

22. Nguyen M, Herszberg I, Paton R. The shear properties of woven carbon fabric. Compos Struct 1999;47(1-4):767-779.

23. Kelly JM. Generalisation of some elastic-viscoplastic stress-strain relations. T Soc Rheol $1967 ; 11(1): 55-76$ 
24. Sharma SB, Sutcliffe MPF, Chang SH. Characterisation of material properties for draping of dry woven composite material. Compos Part A-Appl S 2003;34(12):1167-1175.

25. Fylstra D, Lasdon L,Watson J, Waren A. Design and use of the Microsoft Excel Solver. Interfaces 1998;28(5):29-55. 